\title{
Development of fuzzy rule-based parameters for urban object-oriented classification using very high resolution imagery
}

\begin{abstract}
Urban areas consist of spectrally and spatially heterogeneous features. Advanced information extraction techniques are needed to handle high resolution imageries in providing detailed information for urban planning applications. This study was conducted to identify a technique that accurately maps impervious and pervious surfaces from WorldView-2 (WV-2) imagery. Supervised per-pixel classification algorithms including Maximum Likelihood and Support Vector Machine (SVM) were utilized to evaluate the capability of spectral-based classifiers to classify urban features. Object-oriented classification was performed using supervised SVM and fuzzy rule-based approach to add spatial and texture attributes to spectral information. Supervised object-oriented SVM achieved $82.80 \%$ overall accuracy which was the better accuracy compared to supervised per-pixel classifiers. Classification based on the proposed fuzzy rule-based system revealed satisfactory output compared to other classification techniques with an overall accuracy of $87.10 \%$ for pervious surfaces and an overall accuracy of $85.19 \%$ for impervious surfaces.
\end{abstract}

Keyword: Object-oriented classification; Fuzzy rule-based classification; Optimum index factor; High resolution image 